\title{
Cistanoside A promotes osteogenesis of primary osteoblasts by alleviating apoptosis and activating autophagy through involvement of the Wnt/B-catenin signal pathway
}

\author{
Tongying Chen ${ }^{1 \#}$, Fenghe Gao ${ }^{1 \#}$, Dan Luo ${ }^{2 \#}$, Shihao Wang ${ }^{1}$, Yu Zhao ${ }^{1}$, Shuhua Liu ${ }^{1}$, Jiachun Huang ${ }^{3}$, \\ Yanping Lin ${ }^{1}$, Zhihai Zhang ${ }^{3}$, Hongxing Huang ${ }^{3}$, Lei Wan ${ }^{3}$ \\ ${ }^{1}$ Guangzhou University of Chinese Medicine, Guangzhou, China; ${ }^{2}$ Research Laboratory of Spine Degenerative Disease, The Second Affiliated \\ Hospital of Guangzhou University of Chinese Medicine, Guangzhou, China; ${ }^{3}$ Department of Osteoporosis, The Third Affiliated Hospital of \\ Guangzhou University of Chinese Medicine, Guangzhou, China \\ Contributions: (I) Conception and design: H Huang, L Wan, T Chen; (II) Administrative support: T Chen, F Gao; (III) Provision of study materials \\ or patients: T Chen, D Luo; (IV) Collection and assembly of data: S Wang, Y Zhao; (V) Data analysis and interpretation: J Huang, Y Lin, S Liu, \\ Z Zhang; (VI) Manuscript writing: All authors; (VII) Final approval of manuscript: All authors. \\ \#These authors contributed equally to this work. \\ Correspondence to: Lei Wan; Hongxing Huang. The Third Affiliated Hospital of Guangzhou University of Chinese Medicine, 261 Longxi Rd., \\ Guangzhou 510378, China. Email: gzwl@gzucm.edu.cn; hhx@gzucm.edu.cn.
}

Background: As a phenylethanoid glycoside extracted from Cistanche deserticola, cistanoside A has been shown to have antioxidative effects. In recent years, it has been found to play an important role in osteoporosis.

Methods: Primary osteoblasts were randomly divided into a cistanoside A (Cis A)-1 group ( $5 \mu \mathrm{M})$, a Cis A-2 group $(10 \mu \mathrm{M})$, and a Cis A-3 group $(20 \mu \mathrm{M})$ to screen the optimal dose. Then, cells were treated with Rapamycin (Rapa), 3-MA, Dickkopf-1 (DKK-1), 3MA + Cis A (10 $\mu \mathrm{M})$, and DKK-1 + Cis A (10 $\mu$ M). After a certain period of routine culture, Alkaline Phosphatase (ALP) and Alizarin Red S Staining were performed again and the cells were collected for subsequent experiments including immunofluorescence staining, western blotting, transmission electron microscopy, mitochondrial membrane measurement, and Annexin- $\mathrm{V}$ Fluorescein isothiocyanate (Annexin-V-FITC).

Results: The optimal Cis A dose that preserved osteoblast viability and activated osteogenesis was $10 \mu \mathrm{M}$. It appeared that Cis A $(10 \mu \mathrm{M})$ decreased apoptosis and augmented autophagy via increasing microtubuleassociated protein light chain 3 (LC3)-I/II expressions as well as raising $W n t / \beta$-catenin signal pathway activity. The addition of 3-MA further inhibited osteogenic differentiation and suppressed $W$ nt $/ \beta$-catenin signal pathway activity to increase apoptosis while reducing autophagy levels. A combination of Cis A and DKK-1 resulted in higher levels of apoptosis but lower levels of autophagy.

Conclusions: Cis A appears to be a potent inducer of autophagy and inhibitor of apoptosis in primary osteoblasts by working through the $\mathrm{Wnt} / \beta$-catenin signal pathway, thereby resulting in enhanced osteogenic differentiation.

Keywords: Cistanoside A (Cis A); apoptosis; autophagy; Wnt/ $\beta$-catenin; osteoporosis; osteoblasts

Submitted Nov 12, 2021. Accepted for publication Jan 07, 2022.

doi: $10.21037 / \mathrm{atm}-21-6742$

View this article at: https://dx.doi.org/10.21037/atm-21-6742 


\section{Introduction}

Osteoporosis is a chronic disease characterized by deterioration of the bone structure and low bone density (1). It is the product of imbalanced bone remodeling, namely the occurrence of excess bone resorption and reduced bone formation. The resultant abnormal bone structure and function leads to heightened bone fragility and risk of fracture (1). In terms of risks of osteoporosis, it is acknowledged age and hereditary, smoking, Vitamin D deficiency, Calcium shortage are the mainly factors (2). The latest research aimed at discovering new treatment methods for osteoporosis has involved pharmacological osteoclast inhibition as it holds a central role in bone resorption (3). Current clinical agents for treating osteoporosis target stimulation of bone formation and/or inhibiting bone resorption, for example, teriparatide, bisphosphonates, and receptor activator of nuclear factor-KB (RANK) ligand inhibitors like dinozumab. Nevertheless, these medications are plagued by poorly tolerated adverse effects such as gastrointestinal side effects, atrial fibrillation, and other cardiovascular events (4). It is imperative that newer, more convenient, and effective treatment modalities are discovered to manage this debilitating disease.

Traditional Chinese medicine (TCM), with a history of over 2,000 years, has widely been shown effective in the management of various diseases, including osteoporosis, and appears to work by reducing bone loss, decreasing bone resorption, and enhancing bone formation (5). The well-established TCM agent Cistanche deserticola, known as the "ginseng of the deserts" has long been heralded for its anti-osteoporotic active compounds which include phenylethanoid glycosides (PhGs), echinacoside (ECH), and acteoside (ACT) $(6,7)$. Among which, A cistanche extract, Cis A, is a type of phenylethanoid glycoside that has been shown to possess antioxidative effects which can inhibit apoptosis by protecting against generation of reactive oxygen species (ROS) $(8,9)$. Its therapeutic effect function on bone metabolism by regulating osteoclasts through phosphatidylinositol 3 kinase (PI3K)/ protein kinase B (AKT) pathway and its participation on autophagy procedure via Receptor activator of NF- $\kappa \mathrm{B}$ ligand (RANKL)/RANK/TNF receptor associated factor 6 (TRAF6) pathway have been proven, while its function on osteoblast remains unknown (10). Based on this knowledge, we hypothesized that Cis A may potentially influence osteoblast activity.

Apoptosis is a highly regulated programmed metabolic process which impairs the balance of osteogenesis, resulting in the deterioration of bone. Autophagy is a process involving programmed cell death and is part of the cellular energy management system, development, and cellular stress response (11). Autophagy appears to be associated in osteocyte, osteoblast, and osteoclast differentiation, highlighting its role in bone homeostasis and bone marrow disease $(12,13)$. The relationship between apoptosis and autophagy has not been illustrated clearly yet, some have argued that apoptosis and autophagy would appear at the same time, while others have asserted that autophagy inhibits apoptosis. The $\mathrm{Wnt} / \beta$-catenin pathway is critical for osteoblasts and osteogenic matrix formation (14-16). The accumulation of $\beta$-catenin is vital for activation of the Wnt/ $\beta$-catenin signaling pathway (17) and contributes to osteoblasts development and bone formation (18), while Gsk-3 $\beta$ is a negative factor which stabilizes $\beta$-catenin (19). However, the relationship between autophagy and the Wnt/ $\beta$-catenin pathway in osteoblasts has remained unknown, let alone the relation among $\mathrm{Cis} \mathrm{A}$, the $\mathrm{Wnt} / \beta$-catenin pathway, and autophagy as well as apoptosis.

In the following series of experiments, we documented the role of Cis A in osteogenic differentiation and mineralization ability through apoptosis and autophagy modification via the $\mathrm{Wnt} / \beta$-catenin pathway.

We present the following article in accordance with the ARRIVE reporting checklist (available at https://atm. amegroups.com/article/view/10.21037/atm-21-6742/rc).

\section{Methods}

\section{Reagents}

All animal-related protocols were formulated based on the Guide for the Care and Use of Laboratory Animals by the National Research Council and approved by the Animal Care and Use Committee of Guangzhou University (No. SCXK2018-0034). Cis A (purity $\geq 98 \%$ ) was purchased from Chengdu Must Bio-Technology Co., Ltd. (Chengdu, China); Rapamycin (Rapa) was purchased from Selleck (Houston, TX, USA). The 3-MA was purchased from MedChemExpress (Monmouth Junction, NJ, USA); Dkk-1 protein was purchased from Sino Biological (Beijing, China); the rabbit LC3 antibody, Sequestosome 1 (p62), Beclin-1, Osteopontin (OPN), Runx family transcription factor 2 (Runx2), Osteocalcin (OCN), Bone morphogenetic

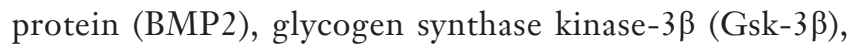
$\beta$-catenin, and glyceraldehyde 3 -phosphate dehydrogenase 
(GAPDH) antibodies were obtained from Cell Signaling Technology (CST; Danvers, MA, USA). Life Technologies (Carlsbad, CA, USA) supplied 4',6-diamidino-2phenylindole (DAPI), Cyanine 3, DyLight 488, and Goat anti-mouse $\operatorname{IgG}(\mathrm{H}+\mathrm{L})$ cross-adsorbed secondary antibodies. Cell Counting Kit-8 (CCK-8) was purchased from Bio sharp (Guangzhou, China); alkaline phosphatase (ALP) activity detection kit and the ALP kit were purchased from Beyotime Biotechnology (Shanghai, China); Alizarin Red S Staining Kit was purchased from Nanjing Jiancheng Bioengineering (Nanjing, China); and dimethyl sulfoxide (DMSO) and fetal bovine serum (FBS) were purchased from Sigma (Sigma-Aldrich, St. Louis, MO, USA). We obtained $\alpha$-Modified Eagle's Medium ( $\alpha$-MEM) and penicillin/ streptomycin from Gibco-BRL (Grand Island, NY, USA) and Hyclone (Thermo-Fisher Scientific, Waltham, MA, USA), respectively.

\section{Cell culture}

\section{Cell isolation and cell culture}

We procured 2-day-old Sprague-Dawley (SD) rats from the Laboratory Animal Center at Guangzhou University of Chinese Medicine (SCXK2018-0034). Cranial osteoblasts (OBs) were extracted by removing rat calvarial connective tissue and placing cleaned skulls in phosphate-buffered saline (PBS). Calvarias were then cut into several small fragments $(2 \times 2 \mathrm{~mm})$ and subjected to ethylenediamine tetraacetic acid (EDTA) enzymatic digestion for $15 \mathrm{~min}$, followed by $0.1 \%$ collagenase-II for $30 \mathrm{~min}$ at $37{ }^{\circ} \mathrm{C}$. Cells were centrifuged to collect $\mathrm{OB}$ cells which were resuspended in $\alpha$-MEM. The isolated primary OBs were cultured with $\alpha$-MEM supplemented with $10 \%$ FBS, $2 \mathrm{mM} \mathrm{L}$-glutamine, $100 \mathrm{U} / \mathrm{mL}$ penicillin, and $100 \mu \mathrm{g} / \mathrm{mL}$ streptomycin at $37{ }^{\circ} \mathrm{C}$ in a humidified atmosphere of $5 \%$ $\mathrm{CO}_{2}$ for $7 \mathrm{~d}$. Osteoblast differentiation and mineralization was induced using osteogenic induction medium (OIM; comprising $1 \% \beta$-glycerophosphate, $0.01 \%$ dexamethasone, and $0.2 \%$ ascorbic acid. Media was changed every $24 \mathrm{~h}$.

\section{Cell viability assay}

A CCK-8 assay was used to determine cell viability. A 96well plate was used to house $\mathrm{OB}$ cells $\left(1 \times 10^{3}\right.$ cells/well) prior to Cis $\mathrm{A}(0,5,10,20,40,80$, and $160 \mu \mathrm{M})$ treatment for 24, 72, and $120 \mathrm{~h}$. Each well then received $10 \mu \mathrm{L}$ CCK-8 solution which was then allowed to incubate in a humidified atmosphere of $95 \%$ air and $5 \% \mathrm{CO}_{2}$ for $1 \mathrm{~h}$ at $37{ }^{\circ} \mathrm{C}$. The optical density (OD) was interpreted at $450 \mathrm{~nm}$ though a microplate reader (Bio-Rad Laboratories Inc., Hercules, CA, USA).

\section{ALP activity and staining}

After primary OBs were treated with or without different concentrations of Cis A, Rapa, 3-MA, DKK-1, 3MA + Cis A, and DKK-1 + Cis A for 7 days, ALP activity and ALP staining were detected, respectively. Total protein from cells was isolated at day 7 , bicinchoninic (BCA) protein assay (Beyotime) was utilized to calculate protein concentration of each group. An ALP activity detection kit (Beyotime) was used to measure ALP activity. When it came to ALP staining, the cells were rinsed twice with PBS and fixed with $70 \%$ paraformaldehyde for $30 \mathrm{~min}$. An ALP buffer $(0.15 \mathrm{M}$ $\mathrm{NaCl}, 0.15 \mathrm{M}$ Tris-HCl, $1 \mathrm{mM} \mathrm{MgCl}_{2}$, $\mathrm{pH}$ 9.0) was used to equilibrate cells twice prior to $1 \mathrm{~h}$ incubation with ALP substrate solution (5 L BCIP and $10 \mathrm{~L} \mathrm{NBT}$ in $1 \mathrm{~mL}$ ALP buffer) at $37{ }^{\circ} \mathrm{C}$ in the dark. Distilled water was used to halt the reaction and plates were dried and imaged.

\section{Alizarin red S staining}

Cells were plated onto 6 -well plates at a density of $2 \times 10^{3}$ cells/well and stimulated with or without Cis A, Rapa, 3-MA, DKK-1, 3MA +Cis A, and DKK-1 + Cis A for 2 weeks. Alizarin red $\mathrm{S}$ staining was then used to evaluate the amount of calcium deposits through image interpretation of absorbance measurement at $570 \mathrm{~nm}$ using an enzyme-linked immunosorbent assay (ELISA) reader.

\section{Immunofluorescence staining}

We used 4\% paraformaldehyde (Solarbio, Beijing, China) to fix cells at room temperature for $15 \mathrm{~min}$ before they were rinsed once with $1 \times$ PBS, permeabilized, and blocked (Solarbio, Beijing, China) for $10 \mathrm{~min}$ at $37^{\circ} \mathrm{C}$, and then incubated overnight at $4{ }^{\circ} \mathrm{C}$ with LC3 antibody (D3U4C; CST, USA). This was followed by a $1 \mathrm{~h}$ incubation period with a fluorescent secondary antibody (1:200 dilution) at $37^{\circ} \mathrm{C}$. The cells were then rinsed once with PBS, exposed to DAPI $(1: 1,000)$ (Sigma, USA) for $10 \mathrm{~min}$, and then covered with glycerin.

\section{Mitochondrial membrane measurement}

We mixed $50 \mu \mathrm{L} \mathrm{JC}-1(200 \mathrm{x})$ and $8 \mathrm{~mL}$ ultrapure water well, then $2 \mathrm{~mL} \mathrm{JC}-1$ dyeing buffer $(5 x)$ was added and mixed 
well were to form JC-1 dyeing working solution. We added $1 \mathrm{~mL} \mathrm{JC}-1$ dye buffer $(5 x)$ to every $4 \mathrm{~mL}$ distilled water and a JC-1 dyeing buffer $(1 \times)$ ice bath was applied. After 5 days of culture, the cells were counted by cell counter, and the number of cells was adjusted to $1 \times 10^{6}$ cells $/ \mathrm{mL}$. After $20 \mathrm{~min}$ incubation at $37^{\circ} \mathrm{C}$, the cells were centrifuged at 2,300 $\mathrm{rpm}$ for $3 \mathrm{~min}$ at $4^{\circ} \mathrm{C}$ to precipitate. The supernatant was discarded and washed twice with JC-1 staining buffer $(1 \times)$. Then, $1 \mathrm{~mL} \mathrm{JC-1}$ staining buffer $(1 \times)$ was added to resuspend the supernatant and detected by up flow cytometry. In normal cells, the mitochondrial membrane potential was high, the concentration of JC-1 aggregates in mitochondria was high, and the red fluorescence was enhanced; when the cells were damaged, the mitochondrial membrane potential decreased, and JC-1 in mitochondria was in the form of monomer, which emitted green fluorescence. Therefore, when the green fluorescence was enhanced or the red fluorescence was decreased, it meant that the mitochondrial membrane potential had decreased and the mitochondria were damaged.

\section{Apoptosis analysis}

After 5 days of culture, cells were collected and stained with an Annexin-VFITC kit for cell apoptosis detection (SigmaAldrich). The apoptosis of the cells was detected by flow cytometry using an excitation wavelength of $488 \mathrm{~nm}$ and an emission wavelength of $525 \mathrm{~nm}$ [for the detection of propidium iodide $(\mathrm{PI})]$.

\section{Western blot assay}

Radioimmunoprecipitation assay (RIPA) lysis buffer (Beyotime Biotechnology, Shanghai, China) supplemented with protease inhibitors (Roche, Basel, Switzerland) was used to lyse proteins in treated OBs. Total protein quantification was carried out using the BCA Protein Assay Kit (Pierce Manufacturing Inc., Appleton, WI, USA). A $10 \%$ sodium dodecyl sulphate-polyacrylamide gel electrophoresis (SDS-PAGE) was first used to separate protein samples. Proteins were then blotted onto polyvinylidene fluoride (PVDF) membranes. Primary antibodies of LC3A/B (D3U4C), Beclin-1 (D40C5), Anti-P62 (SQSTM1) pAb (P62, PM045), GSK-3 $\beta$ (D5C5Z), $\beta$-Catenin (D10A8), GAPDH (14C10) (all from CST, USA), Anti-RUNX2 (ab23981) (Abcam, USA) were incubated with the PVDF membranes at $4{ }^{\circ} \mathrm{C}$ overnight. The second antibody of anti-rabbit [horseradish peroxidase (HRP)]-linked Antibody (7074S, CST) was added the following morning and left to incubate for $1 \mathrm{~h}$ at room temperature. An enhanced chemiluminescence kit (ECL; Thermo Fisher Scientific, Inc., USA) allowed for visualization of the protein bands. The Image Lab Software (Bio-Rad Laboratories Inc., USA) was used to quantify band image intensity.

\section{Transmission electron microscopy}

We used $100 \mathrm{~mm}$ dishes to seed cells at a density of $1.2 \times 10^{5} / \mathrm{mL}$. Cells were cultured for $24 \mathrm{~h}$ and allowed to achieve confluence. Following treatment with or without Cis A, Rapa, 3-MA, DKK-1, 3MA + Cis A, and DKK-1 + Cis A for $5 \mathrm{~d}$, cells were centrifuged for $5 \mathrm{~min}$ in ice-cold PBS at 3,000 rpm, fixed in $500 \mu \mathrm{L}$ of $2.5 \%$ phosphatebuffered glutaraldehyde, followed by final exposure to $1 \%$ osmium tetroxide in the same buffer. A transmission electron microscope was used to visualize cells.

\section{Statistical analysis}

All data were depicted in terms of mean (SD). Statistical analyses were performed using the statistical software SPSS 20.0 (IBM Corp., Armonk, NY, USA). One-way analysis of variance (ANOVA) was used to determine $\mathrm{P}$ values. A $P$ value of less than 0.05 was interpreted as statistically significant.

\section{Results}

\section{Effect of Cis A on primary osteoblast proliferation}

Cis $\mathrm{A}$ is a type of phenylethanoid glycoside (Figure 1A). The primary osteoblasts were isolated from the calvarias of SD rats and cultured to 3 generations (Figure 1B). The effect of Cis A on primary osteoblast proliferation was measured by CCK-8 assay upon exposure to various concentrations of the substrate $(0,5,10,20,40,80$, and $160 \mu \mathrm{M})$ for various durations of time $(1-5 \mathrm{~d})$. As shown in Figure $1 C$, there appeared to be no marked differences between the Cis A $(0,5,10$, and $20 \mu \mathrm{M})$ treatment and the control groups, suggesting that these concentrations did not promote primary osteoblast proliferation. However, at 40, 80, and $160 \mu \mathrm{M}$ Cis A elicited cytotoxicity in primary osteoblasts after 3 and 5 days of incubation (Figure 1C). 

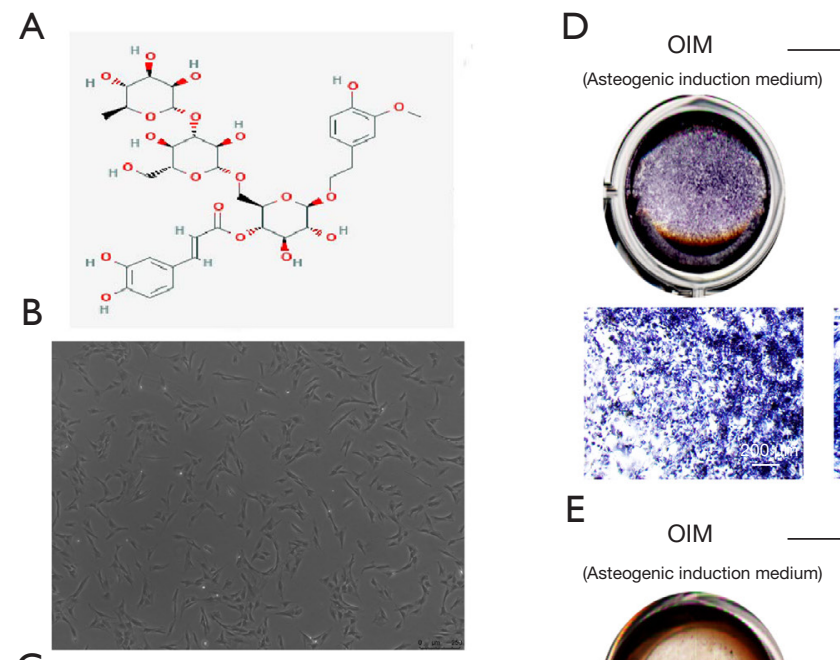

E
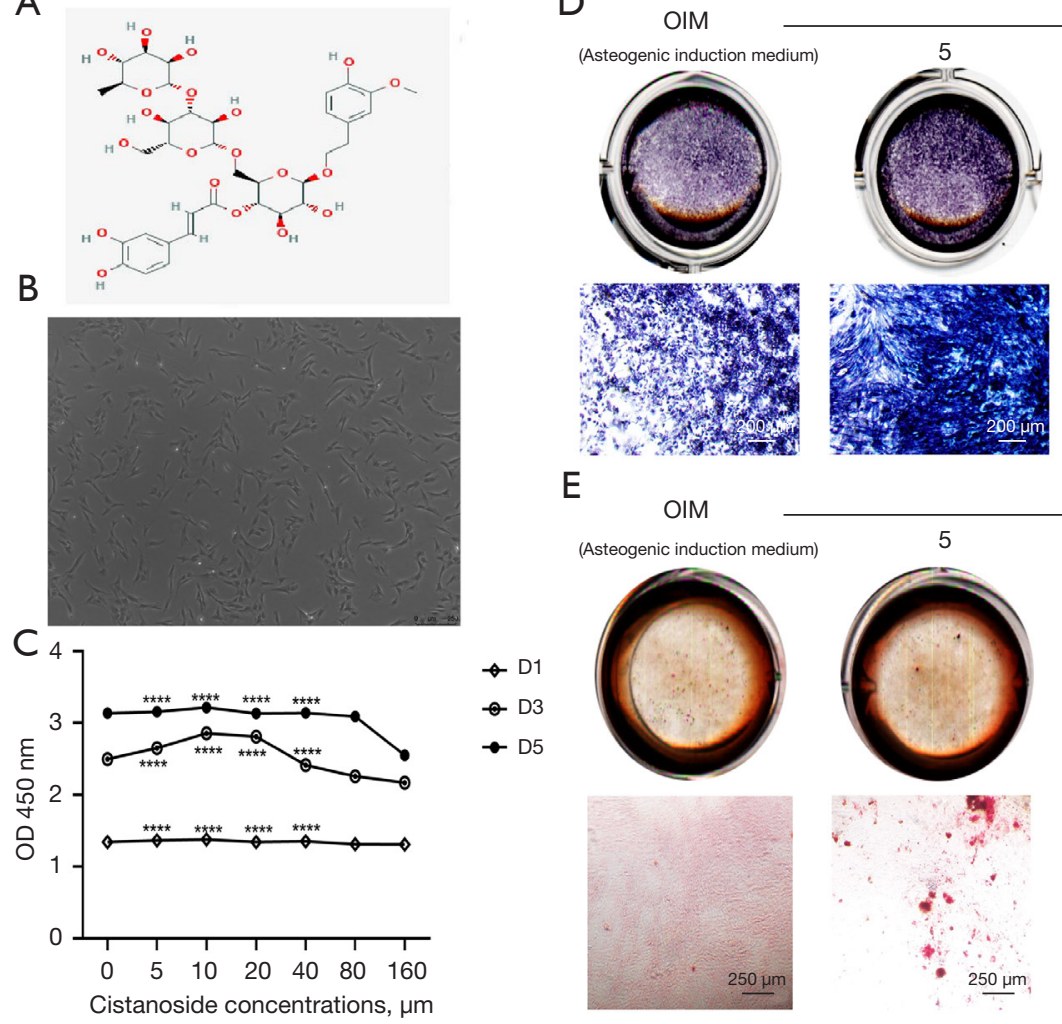

$\mathrm{OIM}+\mathrm{Cis}, \mu \mathrm{m}$

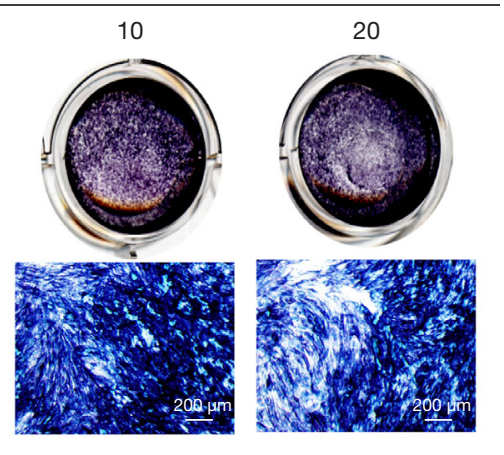

$\mathrm{OIM}+\mathrm{Cis}, \mu \mathrm{m}$
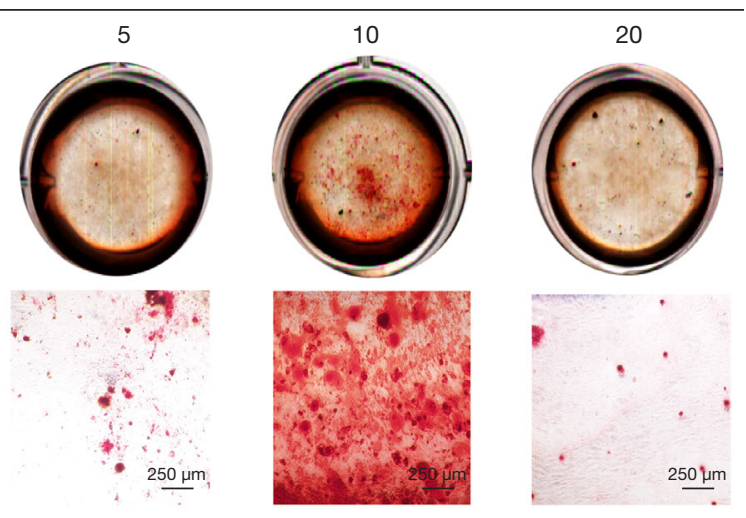

Figure 1 Effects of Cis A on the primary osteoblast proliferation and differentiation as well as mineralization. (A) The structure of Cis A (cite from pubchem). (B) Morphology of the primary osteoblast. (C) Viability of the primary osteoblast treated by Cis A at different concentrations after 1,3 , and $5 \mathrm{~d}$ of culture were measured with CCK- 8 assay. The experiment was repeated 3 times, ${ }^{* * * *}, \mathrm{P}<0.0001$. The cells were cultured with Cis A (5, 10, $20 \mu \mathrm{M})$ for 1, 3, and 7 days. (D) The ALP staining of the primary osteoblasts was measured after incubating with varying concentrations of Cis A for 7 days. Scale bar $=200 \mu \mathrm{m}$. The primary osteoblasts were treated with Cis A $(5-20 \mu M)$ for 14 days. (E) The nodules were stained using Alizarin Red S. Scale bar $=250 \mu \mathrm{m}$. Cis A, Cistanoside A; CCK-8, Cell Counting Kit-8; ALP, alkaline phosphatase.

\section{Cis $A$ accelerates ALP activity and mineralization of primary osteoblasts}

Early osteogenesis is markedly characterized by increased ALP staining, and the formation of calcium nodules by using alizarin red $\mathrm{S}$ staining is a sign of terminal osteogenesis. The optimal concentration of Cis A that promotes primary osteoblast differentiation and mineralization was determined by ALP staining and Alizarin red staining, respectively, following treatment of the cells with 5, 10, or $20 \mu \mathrm{M}$ Cis A for 7 days for ALP staining and 14 days for Alizarin red S staining. Treatment with $10 \mu \mathrm{M}$ Cis A increased ALP expression, while other concentrations were notably less effective. Consistent with results of ALP staining, primary osteoblasts treated with Cis $\mathrm{A}(10 \mu \mathrm{M})$ for 14 days exhibited a significant increase in mineralization in contrast to the control group (Figure 1D,1E).

\section{Inhibition of autophagy reduces osteoblast differentiation and mineralization as well as osteogenesis}

The effect of autophagy on primary osteoblast proliferation was measured by ALP activity, ALP staining, and alizarin red staining following the same treatment protocols as mentioned in ALP activity and staining. Treatment with 3-MA for $7 \mathrm{~d}$ markedly reduced ALP activity and expression. Similar findings were noted with the Alizarin red $\mathrm{S}$ assay (Figure 2A-2C). Furthermore, the measurement of RUNX2 BMP2, OPN, and OCN by western blotting was degraded by 3-MA (Figure 2D-2H). Both RUNX2 and BMP2 are triggers of osteoblast differentiation. OPN and 
A

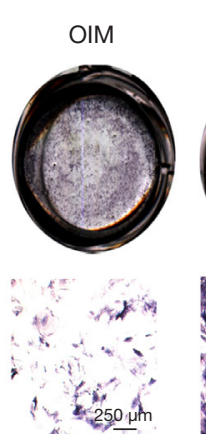

B

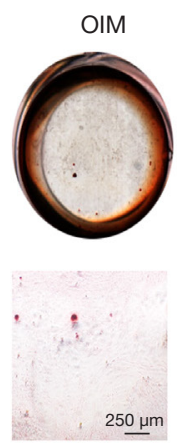

E

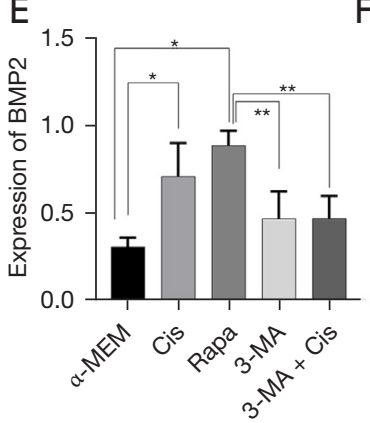

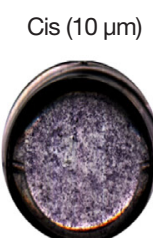
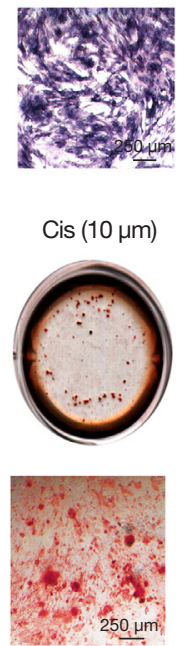

$\mathrm{F}$
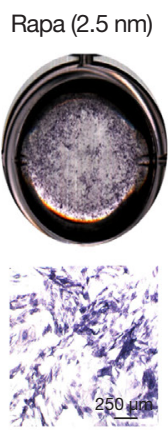

Rapa $(2.5 \mathrm{~nm})$
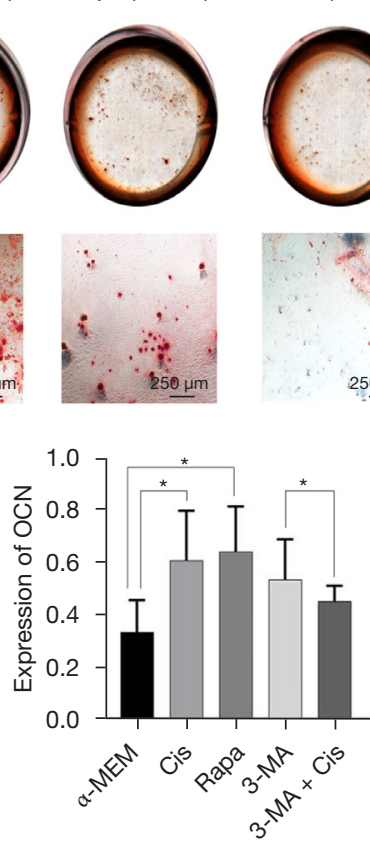

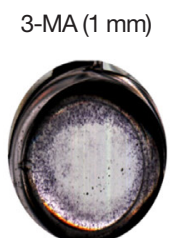

3-MA (1 mm)+

Cis $(10 \mu \mathrm{m})$
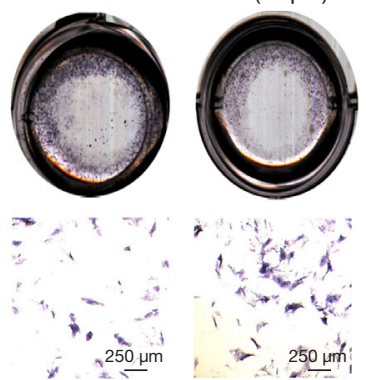

3-MA (1 mm)

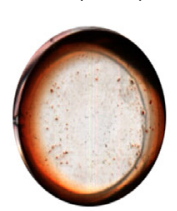

3-MA (1 mm)+ Cis $(10 \mu \mathrm{m})$
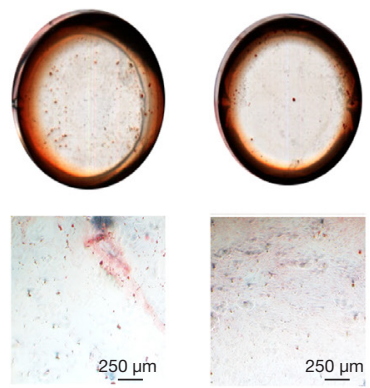

G

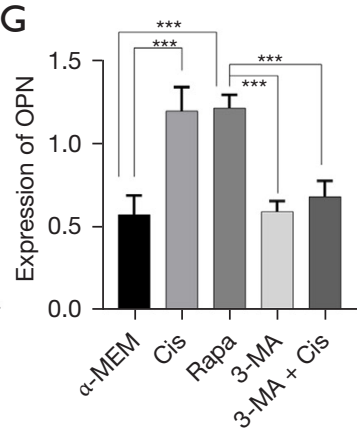

C
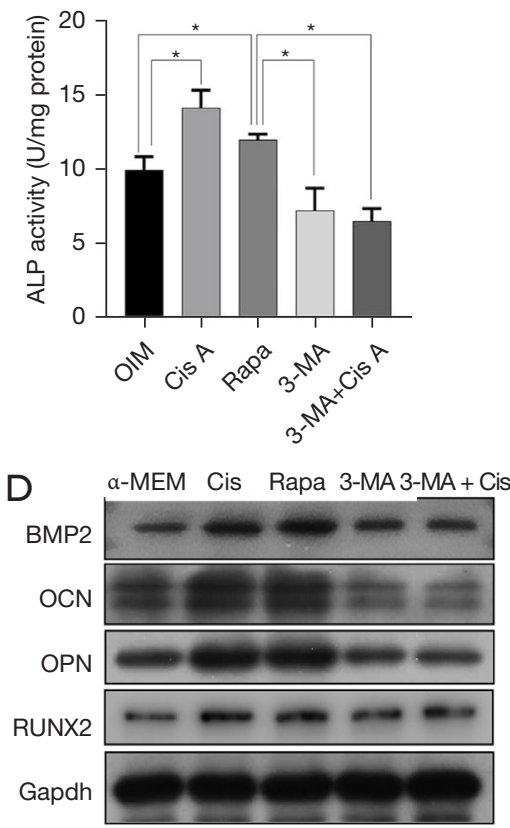

$\mathrm{H}$

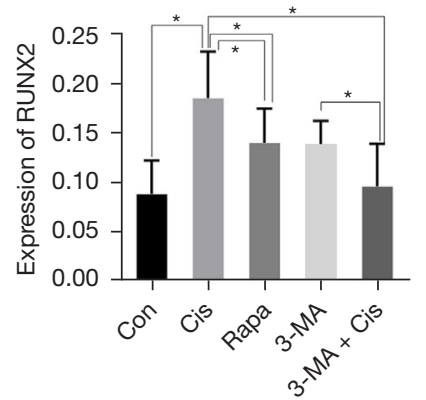

Figure 2 Cis A activates osteogenesis while inhibition of autophagy reduces the differentiation and mineralization of osteoblast. (A) The primary osteoblasts were respectively incubated with Cis A $(10 \mu \mathrm{M})$, Rapa $(2.5 \mathrm{nM})$, 3-MA $(1 \mathrm{mM})$, 3-MA $(1 \mathrm{mM})+$ Cis A $(10 \mu M)$ for 7 days, then the ALP staining of the primary osteoblast was measured. (B) The primary osteoblasts were respectively incubated with OIM, Cis A $(10 \mu \mathrm{M})$, Rapa $(2.5 \mathrm{nM})$, 3-MA $(1 \mathrm{mM})$, 3-MA $(1 \mathrm{mM})$ + Cis A $(10 \mu \mathrm{M})$, then, Alizarin Red S was used to stain the mineralized nodules. Scale bar $=250 \mu \mathrm{m}$. (C) The primary osteoblasts were respectively incubated with Cis A (10 $\mu \mathrm{M})$, Rapa (2.5 nM), 3-MA (1 mM), 3-MA $(1 \mathrm{mM})+$ Cis A $(10 \mu \mathrm{M})$ for 7 days, then the ALP activity was measured. (D) Relative BMP2, OPN, OCN, and RUNX2 protein levels in each group. (E-H) Quantification of relative protein levels. ${ }^{* * *}, \mathrm{P}<0.001,{ }^{* *}, \mathrm{P}<0.01,{ }^{*}, \mathrm{P}<0.05$. Cis A, Cistanoside A; OIM, osteogenic induction medium.

OCN are also pivotal factors of osteogenesis, the expression of RUNX2, BMP2, OPN, and OCN detected by western blotting was elevated by Cis A, while the addition of 3-MA downregulated the levels of the above protein. These results highlight that the restraint of autophagy appeared to inhibit primary osteoblast differentiation and mineralization.

\section{Cis A upregulates Beclin1 and LC3 expressions while promoting autophagosome formation}

Cis A-induced autophagy in osteoblasts was evaluated by intensity of LC3 by immunofluorescence staining and confirmation of autophagosome formation was imaged 


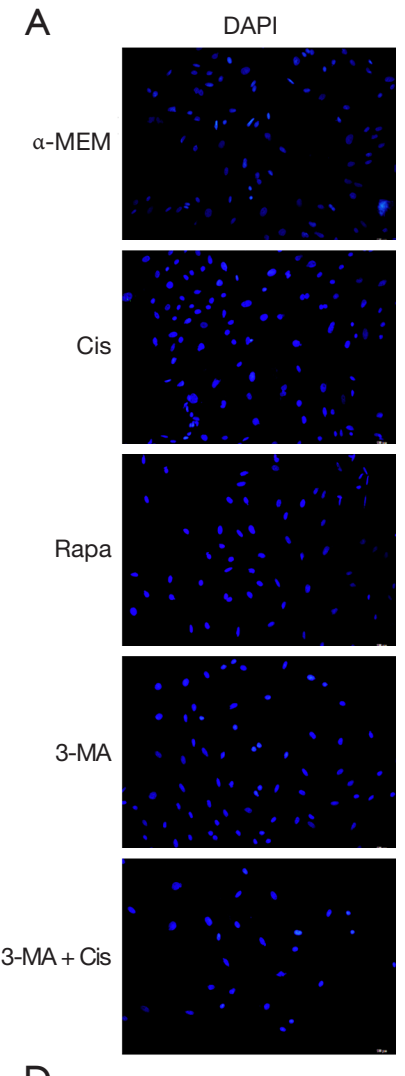

D

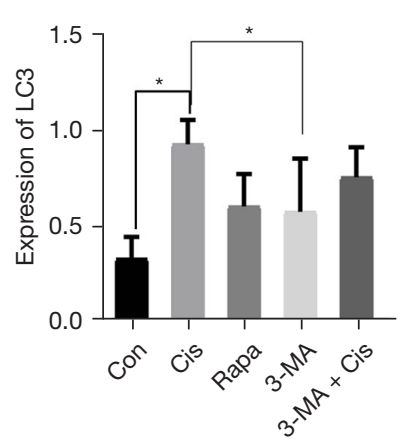

LC3
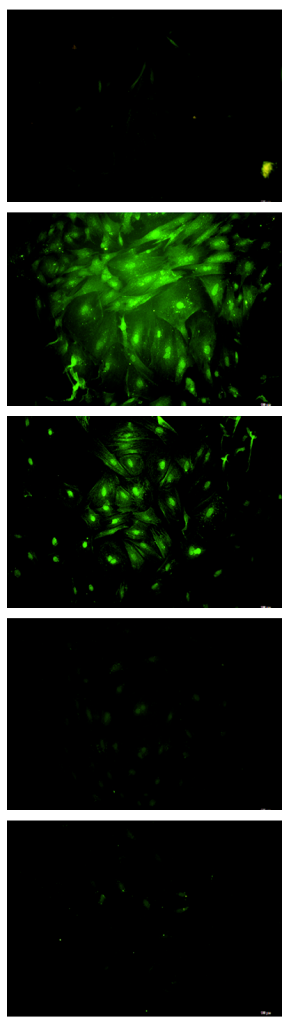

E
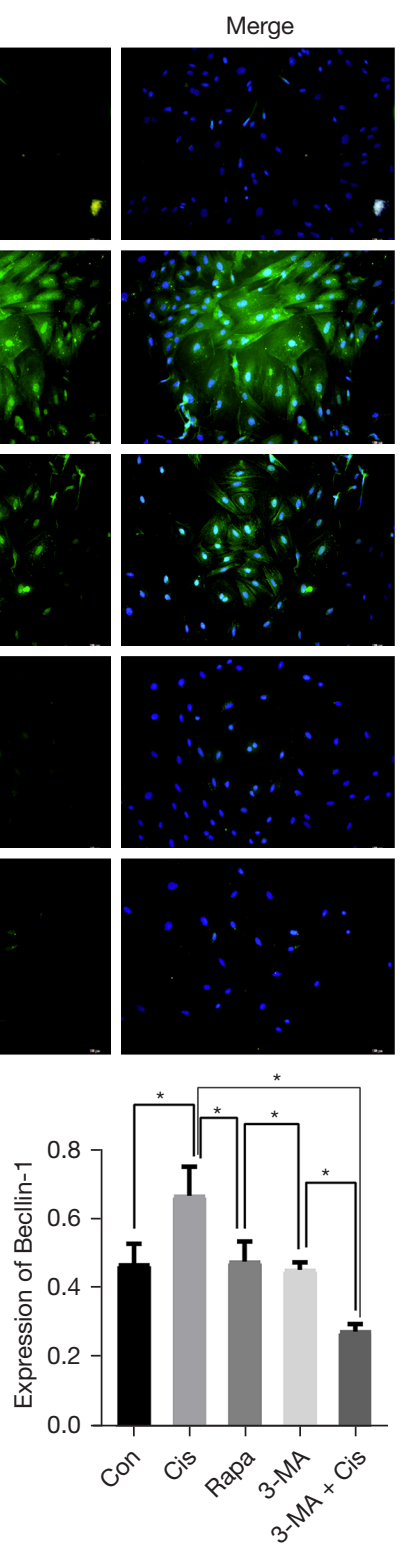

B

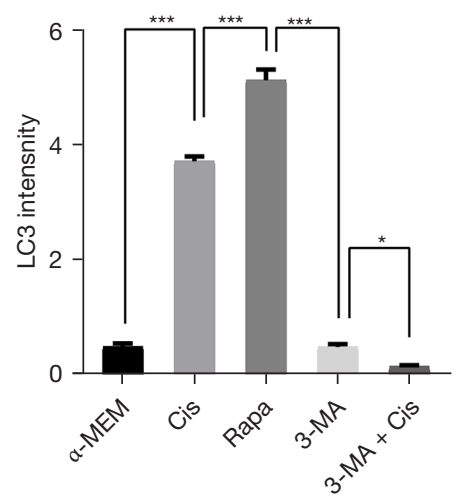

C

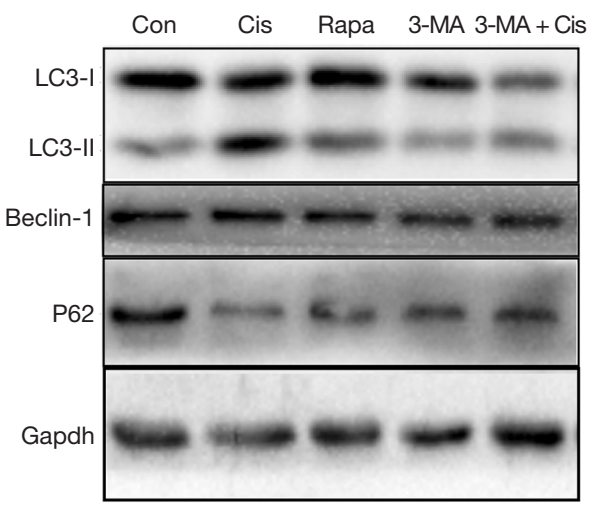

$\mathrm{F}$

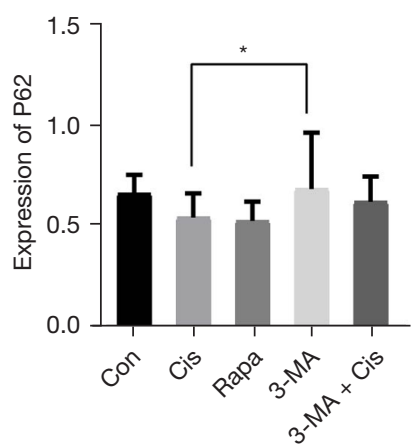

Figure 3 Cis A induces autophagy of the primary osteoblast. (A) Immunofluorescence images of LC3 showing the primary osteoblasts in the Cis A $(10 \mu M)$, Rapa $(2.5 \mathrm{nM}), 3-\mathrm{MA}(1 \mathrm{mM}), 3-\mathrm{MA}(1 \mathrm{mM})+$ Cis A $(10 \mu \mathrm{M})$ group (scale bar $=100 \mu \mathrm{m})$. The green light indicates the LC3 protein. (B) Quantification of LC3's fluorescent intensity. ${ }^{* *}, \mathrm{P}<0.001$. (C) Relative LC3, P62, and Beclin-1 protein levels in each group. (D-F) Quantification of relative protein levels. *, $\mathrm{P}<0.05$. Cis A, Cistanoside A.

using a transmission electron microscope. It is known that 3-MA blocks formation of autophagosomes while rapamycin is a known inhibitor of the mammalian target of rapamycin. Cells were exposed to Cis A $(10 \mu \mathrm{M})$, autophagy inhibitor 3-MA (1 mM), and autophagy inducer rapamycin $(2.5 \mathrm{nM})$ for $5 \mathrm{~d}$. Rapamycin was used as a positive control for induction of primary osteoblast autophagy.
The treatment with Cis A was detected a higher intensity of LC3 (Figure 3A,3B). Expression of Beclin-1 and LC3 II/I, was elevated in primary osteoblasts incubated by Cis A and Rapa cells exposed to 3-MA or 3-MA+Cis A were decreased, while P62 protein negatively unveiled the level of autophagy, highly expressed in cells exposed to 3-MA or $3-\mathrm{MA}+\mathrm{Cis} \mathrm{A}$, but lower in combination treatment of $\mathrm{Cis}$ 


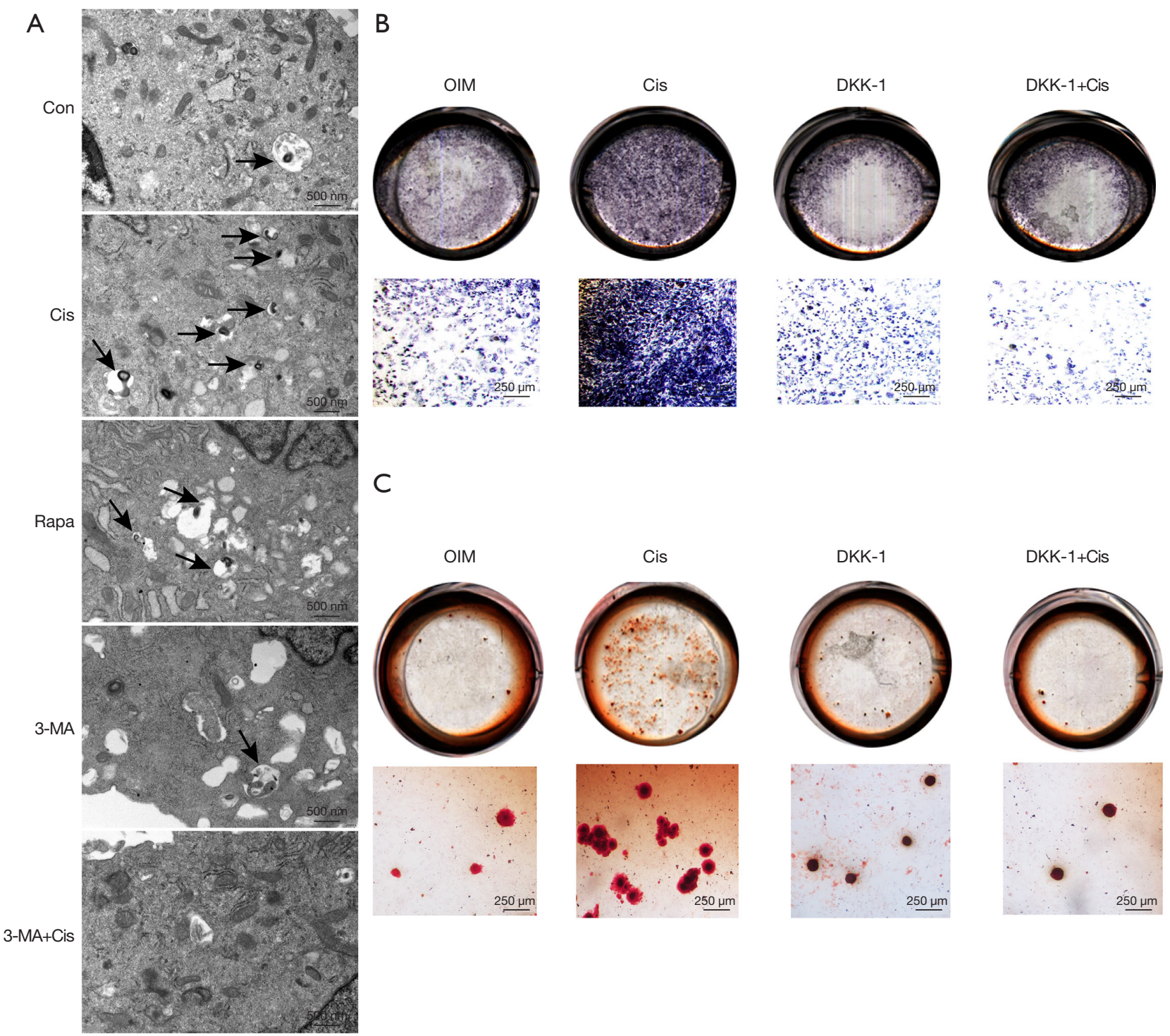

Figure 4 Cis A enhance the formation of autophagosome of the primary osteoblasts and DKK-1 inhibits differentiation and mineralization of the primary osteoblasts. (A) Representative TEM images of the autophagosome incubated with Cis A (10 $\mu M)$, Rapa (2.5 nM), 3-MA $(1 \mathrm{mM}), 3-\mathrm{MA}(1 \mathrm{mM})+$ Cis A $(10 \mu \mathrm{M})$ for $5 \mathrm{~d}$ (scale bar $=500 \mathrm{~nm})$. The black arrowhead pointing at the autophagosome. (B) The primary osteoblasts were respectively incubated with OIM, Cis A $(10 \mu \mathrm{M})$, DKK-1 (100 ng/mL), Cis A $(10 \mu M)+D K K-1(100 \mathrm{ng} / \mathrm{mL})$ for $7 \mathrm{days}$, then the ALP staining of the primary osteoblast was measured. Scale bar $=250 \mu M$. (C) The primary osteoblast were respectively incubated with OIM, Cis A $(10 \mu \mathrm{M})$, DKK-1 $(100 \mathrm{ng} / \mathrm{mL})$, Cis A $(10 \mu \mathrm{M})+\mathrm{DKK}-1(100 \mathrm{ng} / \mathrm{mL})$ for 14 days. Then, Alizarin Red S was used to stain the mineralized nodules. Scale bar $=250 \mu \mathrm{M}$. Cis A, Cistanoside A; TEM, transmission electron microscope; OIM, osteogenic induction medium.

$\mathrm{A}$ and Rapa (Figure 3C-3F). In addition, Cis A treatment appeared to stimulate autophagosome treatment. However, combination treatment of 3-MA with $\mathrm{Cis} \mathrm{A}$ did not result in autophagosome formation (Figure 4A). We therefore concluded that $\mathrm{Cis} \mathrm{A}$ is able to trigger autophagy in osteoblasts, with autophagy in turn activating osteoblast 

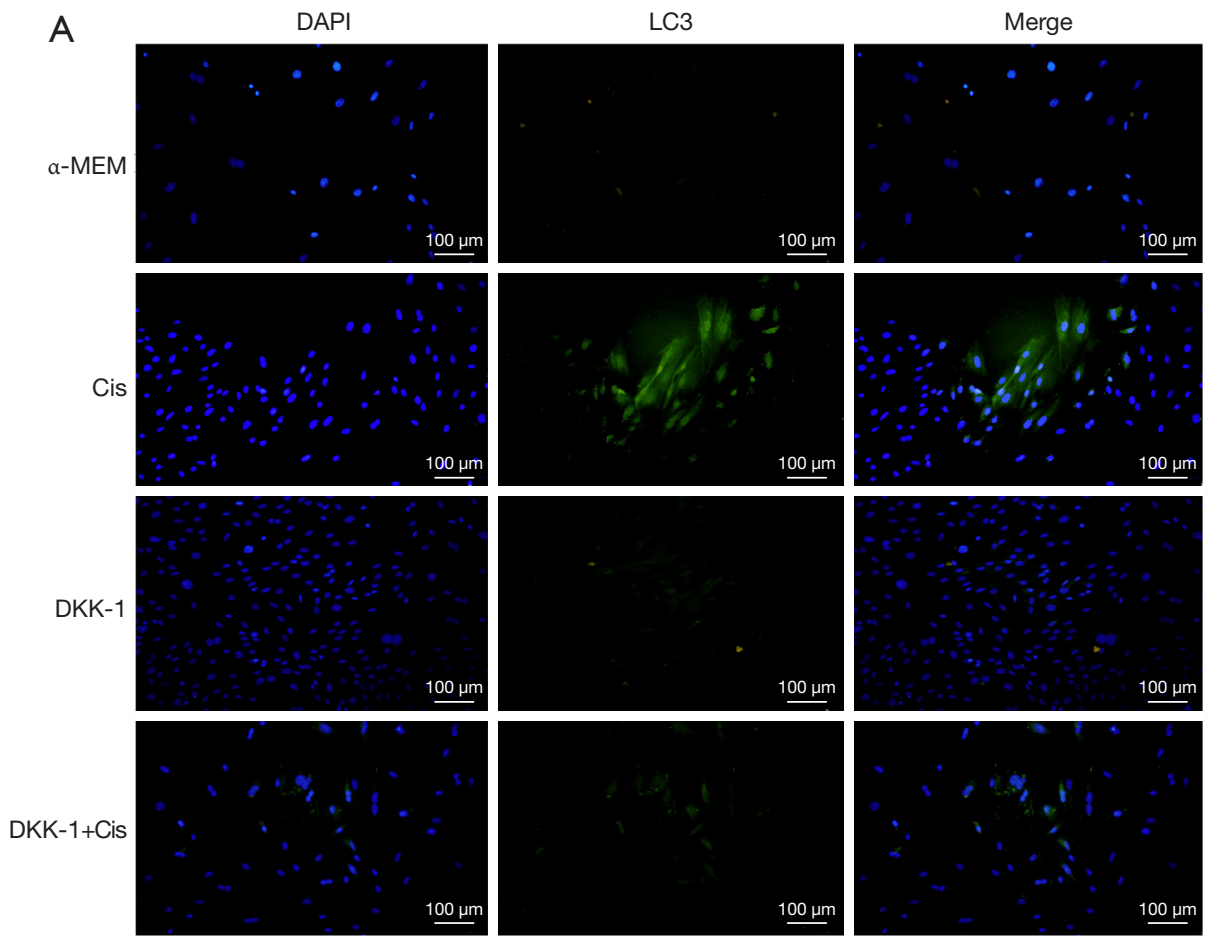

C

a-MEM

Cis
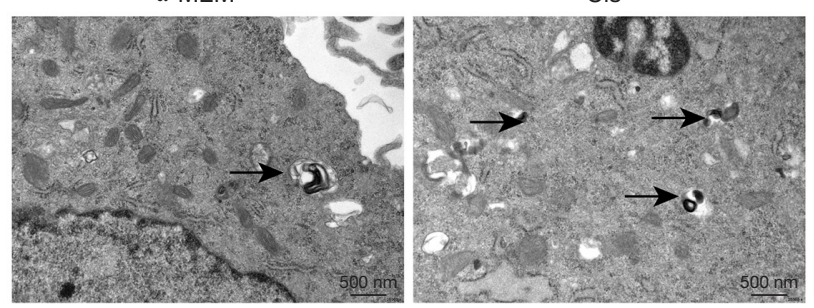

DKK-1

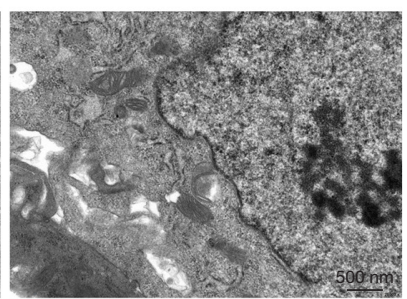

B

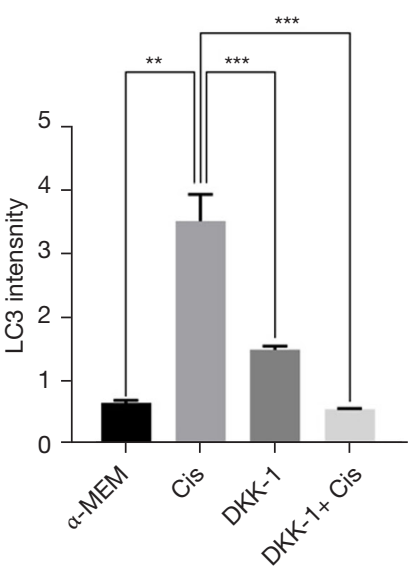

Figure 5 Inhibition of the $W n t / \beta$-catenin pathway represses autophagy of the primary osteoblasts. (A) Immunofluorescence images of LC3 showing the primary osteoblast in the $\alpha-M E M$, Cis A $(10 \mu M)$, DKK-1 (100 ng/mL), Cis A $(10 \mu M)+$ DKK-1 (100 ng/mL) (scale bar $=100 \mu \mathrm{m}$ ). The green light indicates the LC3 protein. (B) Quantification of LC3's fluorescent intensity. (C) Representative TEM images of the autophagosome incubated with $\alpha$-MEM, Cis A $(10 \mu \mathrm{M})$, DKK-1 (100 ng/mL), Cis A $(10 \mu M)+$ DKK-1 (100 ng/mL). The black arrowhead pointing at the autophagosome. Cis $\mathrm{A}$, Cistanoside $\mathrm{A}$; TEM, transmission electron microscope. ${ }^{* * *}, \mathrm{P}<0.001,{ }^{* *}, \mathrm{P}<0.01$.

differentiation.

\section{Cis $A$ induces autophagy in primary osteoblasts through activation of the Wnt/B-catenin pathway}

The Wnt/ $\beta$-catenin pathway is critical for osteoblasts and osteogenic matrix formation. To investigate whether Cis A-induced autophagy in primary osteoblasts involved the $\mathrm{Wnt} / \beta$-catenin pathway, $\mathrm{Wnt} / \beta$-catenin pathway inhibitors such as DKK-1 were used. The degree of osteoblast differentiation and mineralization were evaluated using ALP and Alizarin red S staining kits, transmission electron microscopy, and western blotting. Cells treated with DKK-1 were noted to have reduced degrees of ALP and Alizarin red S staining intensities (Figure $4 B, 4 C$ ), indicating the blocking of $\mathrm{Wnt} / \beta$-catenin pathways inhibited the differentiation and mineralization of the primary osteoblast. We then performed immunofluorescence staining (Figure $5 A, 5 B$ ) and western blotting. The decreasing expression of LC3 both in immunofluorescence staining and western blotting implied that blocking of Wnt/ $\beta$-catenin pathways suppressed autophagy in the primary osteoblasts. When cells were cultured in combination of Cis A and DKK-1, the expression of LC3 was consistent 
A

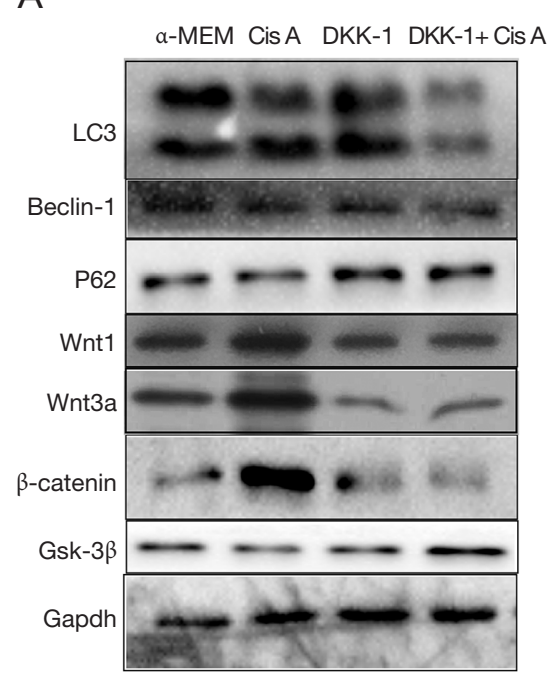

$\mathrm{F}$

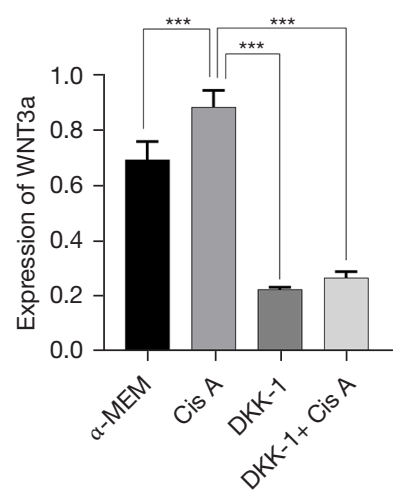

B
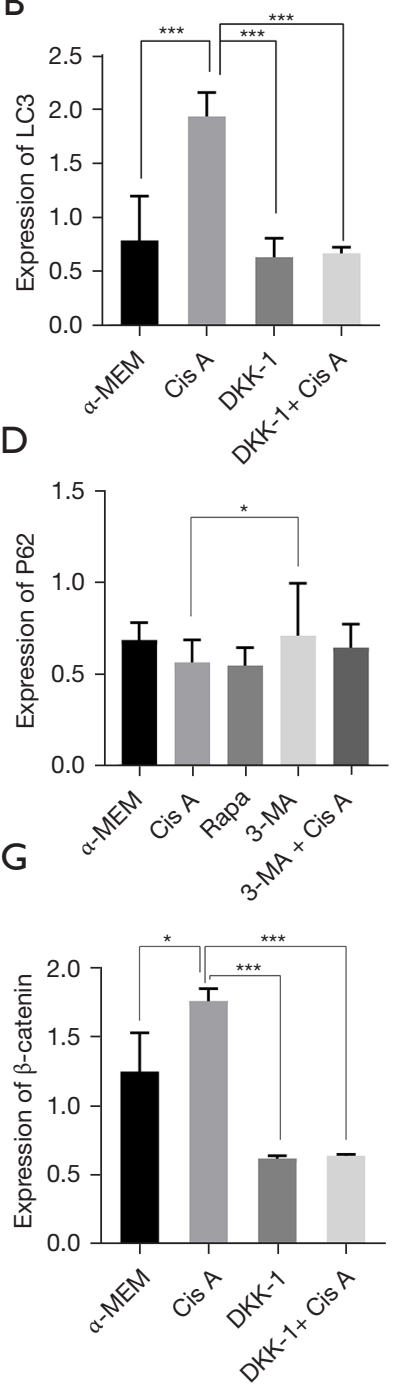

C

$\mathrm{E}$
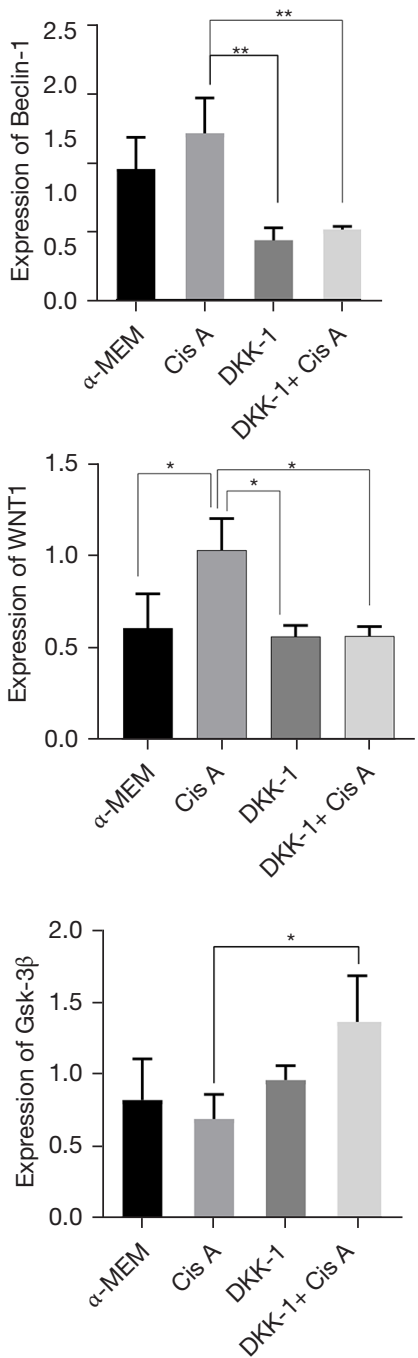

Figure 6 Cis A induces autophagy in primary osteoblast through the Wnt/ $\beta$-catenin pathway. (A) Relative LC3, P62, Beclin-1, WNT1, WNT3a, $\beta$-catenin, and Gsk-3 $\beta$ protein levels in each group. (B-H) Quantification of relative protein levels. ${ }^{* * *}, \mathrm{P}<0.001,{ }^{* *}, \mathrm{P}<0.01,{ }^{*}$, $\mathrm{P}<0.05$. Cis A, Cistanoside A.

with the result that incubated with DKK-1, suggesting that Cis A may induce autophagy via $\mathrm{Wnt} / \beta$-catenin pathways. Furthermore, the trends of results tested in transmission electron were consistent with the above (Figure 5C). Ultimately, Wnt1, Wnt3, Gsk-3 $\beta$, and $\beta$-catenin were tested by western blotting, the results reflect that Cis A regulates the $\mathrm{Wnt} / \beta$-catenin pathway potently. These results clearly demonstrate that blocking of the $\mathrm{Wnt} / \beta$-catenin pathway represses autophagy and Cis A may induce autophagy involved in Wnt/ $\beta$-catenin pathway (Figure 6).

\section{Cis A downregulates the level of apoptosis and upregulates the maintenance of mitochondrial membrane}

The level of apoptosis of primary osteoblasts was measured by Annexin-VFITC following the same treatment protocols as mentioned above. Treatment with 3-MA for $7 \mathrm{~d}$ markedly upregulated the level of apoptosis while Cis A downregulated the level of apoptosis (Figure 7A). The mitochondrial membrane was tested by JC-1. Similar findings were congruous with the level of mitochondrial 
A
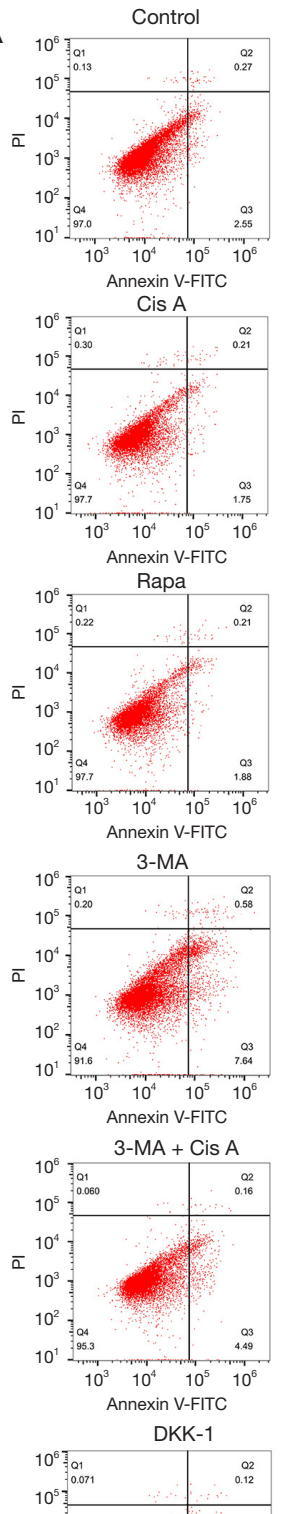

든
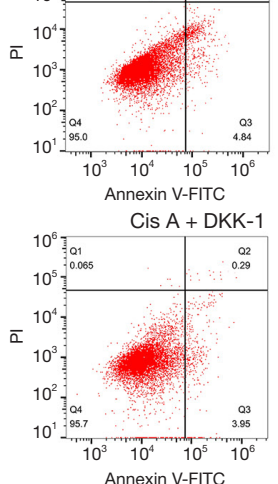

B
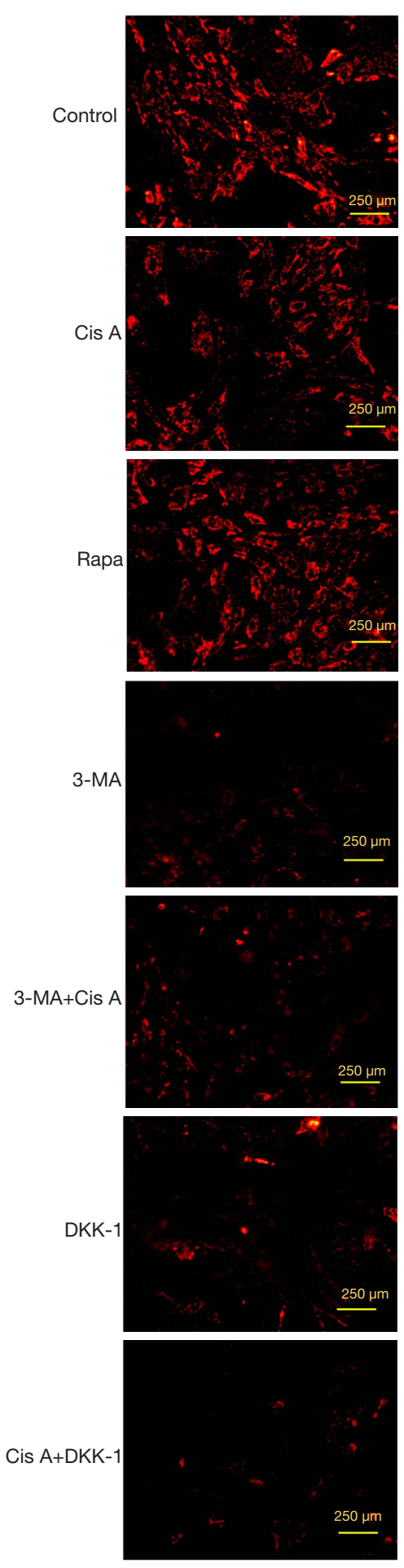

JC-1 aggregates
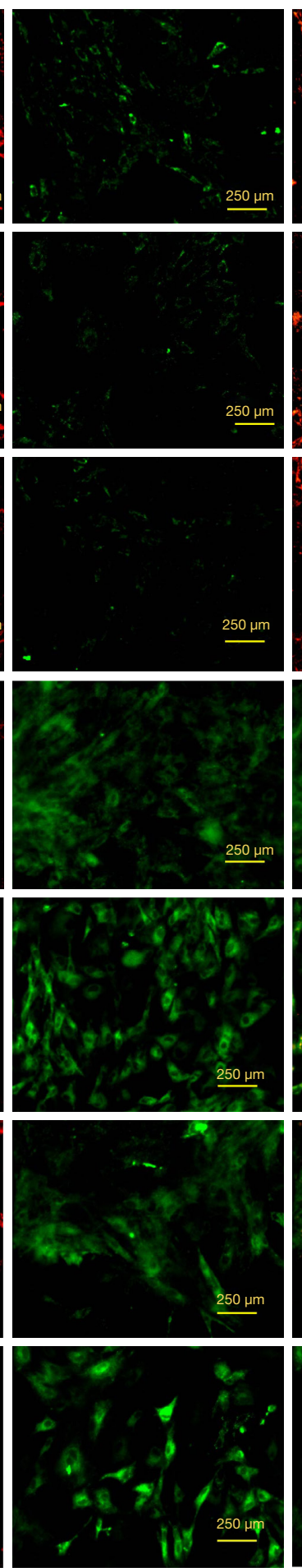

Merge
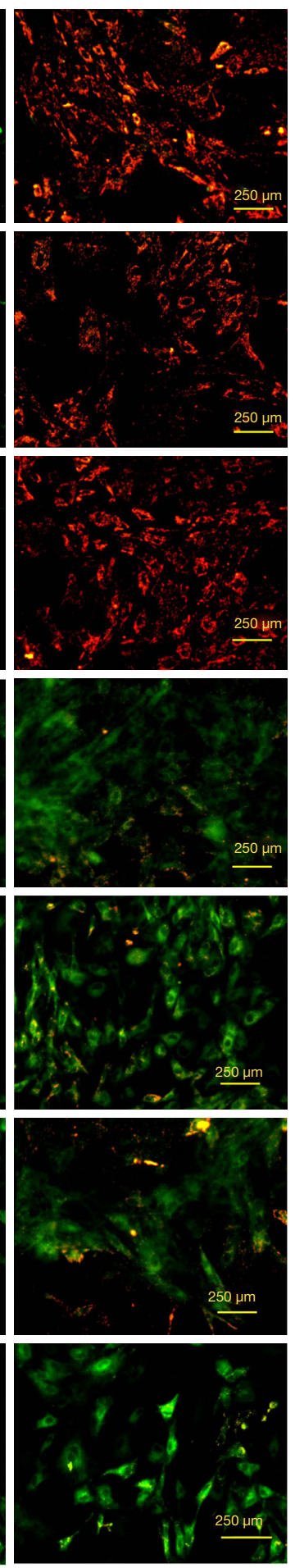

Figure 7 Cis A decreases apoptosis and augment autophagy. (A) Cells were cultured in $\alpha-M E M$, Cis A, Rapa, 3-MA, Cis A + 3-MA, DKK1 and the combination of Cis A and DKK-1. Annexin-VFITC was used to test the apoptosis level of each group. (B) Cells were cultured in a-MEM, Cis A, Rapa, 3-MA, Cis A + 3-MA, DKK-1 and the combination of Cis A and DKK-1. The mitochondrial membrane level was tested by JC-1 (scale bar $=250 \mu \mathrm{m}$ ). 
membrane. These results demonstrated that the Cis A downregulates the level of apoptosis and regulates maintenance of the mitochondrial membrane (Figure $7 B$ ).

\section{Discussion}

Cistanche herba appears to demonstrate anti-osteoporotic properties which are thought to be mediated by its active compounds such as echinacoside, acteoside, and PhG. It has been reported that $\mathrm{PhG}$ possesses anti-oxidant, antiaging, neuroprotective, and osteoblast growth promoting abilities (7). Cis A is a PhG compound and an extract of Cistanches herba. Cis A has been reported to be involved in TRAF6-mediated NF-kappaB inactivation and PI3K/Akt activation in experiments involving OVX mice (10), and it has also been purported to impart benefits in the treatment of neurodegenerative disorders such as vascular dementia, Parkinson's disease, and Alzheimer's disease (20). Moreover, Cis A may also protect against ethanol-induced damage in primary cultured mouse hepatocytes as well as against alcohol-induced hepatotoxicity in mice (21). Our study aimed to investigate the influence of Cis A on osteoblasts. The current investigation demonstrated that Cis A increases osteoblast differentiation and mineralization as evidenced by ALP and Alizarin red S staining. The ALP is a marker of early osteogenic differentiation as well as an indicator of osteoblast activity (22). Raised ALP levels may represent the occurrence of active bone formation (23). Alizarin red S is a chelating agent and its use as a colorimetric regent for cations is well established (24). The increasing expression of osteogenesis protein such as RUNX2, BMP2, OPN, and OCN further demonstrated the activating function of Cis A.

Apoptosis is an essential process for maintenance of homeostasis in multicellular organisms. Osteoblast apoptosis suppresses differentiation and mineralization in vitro by reducing cell density. Our study assessed the function of Cis A on anti-apoptosis with Annexin-VFITC, on account its quality of antioxidation, and revealed that Cis A lowers the level of apoptosis and maintains normal levels of the mitochondrial membrane. Autophagy is another biologically essential component of cellular survival (25). A genomewide association study highlighted the close association of bone mineral density (BMD) and/or osteoporosis with autophagy regulation (26). Autophagy protects osteoblasts through its effects in reducing ROS, inhibiting apoptosis, and regulating mineralization (27). The relationship between osteoblasts and key protein in autophagy can be concluded as follows, osteoblast mineralization has been notably reduced in ATG7 and Beclin1 deficiencies (28). Likewise, an ATG5 deletion decreases in vivo bone volume by decelerating mineralization capacity (29). Osteoblasts which are unable to undergo autophagy experience higher oxidative stress, leading to increased receptor activator of NFKB1 (TNFSF11/RANKL) secretion, thereby leading to bone resorption through increased osteoclast formation (30). When autophagy occurs, autophagosomal membranes express LC3 family proteins, participate in cargo recognition and recruitment, and are cleaved by Atg 4 protease to form LC3 I, which is then activated by Atg7 to yield LC3-II (31). The presence of P62 is a typical signal of autophagy, and when autophagy occurs, the level of P62 will increase. Our study showed a higher expression of LC3II in contrast to LC3 I in Cis A treated groups, suggesting the role of this compound in inducing osteoblast autophagy which in turn is involved in osteoblast mineralization and differentiation. Our study found that Cis A activates autophagy while inhibiting apoptosis, which might provide evidence for the argument that autophagy downregulates apoptosis.

Osteoblasts originate from bone marrow mesenchymal stem cells (BMSCs) and are regulated by the Wnt/ $\beta$-catenin pathway. The Wnt1 signaling proteins consist of Wnt1 family and Wnt5a family proteins (32). The Wnt1 family proteins include $W n t 1, W n t 3 a$, and Wnt7a, which activate the canonical $\mathrm{Wnt} / \beta$-catenin pathway (32), and it help to improve mitochondrial function and prevent apoptotic cell injury (33). The relationship between the $\mathrm{Wnt} / \beta$-catenin pathway and autophagy has yet to be fully documented. In most tumors (34), Wnt/ $\beta$-catenin signaling appears to negatively regulate autophagy, with $\beta$-catenin suppressing levels of p62 (35). Some papers have reported that the $W n t / \beta$-catenin pathway and autophagy can be activated simultaneously, therefore $\mathrm{Wnt} / \beta$-catenin signaling pathway activation was postulated to mediate autophagy in hepatocellular cancer (HCC) cells (36). Other papers have described how the Wnt3a ligand stimulates autophagy through Wnt signaling. In this study, we found that groups treated with Cis A had elevated Wnt1, Wnt3a, and $\beta$-catenin levels with decreased Gsk-3 $\beta$ expressions, indicating that Cis A did activate the $\mathrm{Wnt} / \beta$-catenin pathway. The $\beta$-catenin component is a key protein of the $\mathrm{Wnt} / \beta$-catenin pathway, when the $\mathrm{Wnt} / \beta$-catenin pathway is activated, $\beta$-Catenin will enter the nucleus and affect the next step of gene transcription. Studies have shown that autophagy can increase the expression of $\beta$-Catenin, and then p62 will be inhibited by $\beta$-Catenin. Research has suggested 
that the accumulation of $\beta$-Catenin inhibits the p62 promoter, leading to autophagy inhibition (37). However, our experiment found that the increase of $\beta$-Catenin could decrease the expression of $\mathrm{p} 62$, without inhibiting autophagy; on the contrary, it promoted the occurrence of autophagy. Perhaps this is because $\beta$-catenin accelerated and decreased p62 expression by enhancing autophagy when lysosomal enzymes degraded p62 protein. The Gsk$3 \beta$ is a serine/threonine protein kinase which can inhibit $\beta$-catenin entering the nucleus and inhibit autophagy by phosphorylation of TSC2. The same mechanism is also reflected in Alzheimer's disease, with abnormal Gsk-3 $\beta$ activity having been shown to cause neuronal damage. Our study showed that Cis A downregulates the expression of Gsk-3 $\beta$, which possibly means that Cis A inhibits Gsk-3 $\beta$ expression through the $\mathrm{Wnt} / \beta$-Catenin pathway. Our study also showed that cells treated with DKK-1 only had low levels of autophagy, incongruous with those treated with a combination of DKK-1 and Cis A. Taken together, these findings suggest that Cis A may activate autophagy via the Wnt/ $\beta$-catenin pathway. Base on the results above, Cis A may be potential for development into anti-osteoporosis drugs.

To summarize, we documented that Cis A promotes the differentiation and mineralization of the primary osteoblasts. This process may be mediated through apoptosis and activation of autophagy that involves Wnt/ $\beta$-catenin signal pathway activation.

\section{Acknowledgments}

The authors thank the Laboratory of Osteology and Traumatology of Traditional Chinese Medicine, Lingnan Medical Research Center, Guangzhou University of Chinese Medicine for cell culture and equipment support. Funding: This study was jointly funded by the National Natural Science Foundation of China (81973886), and Projects of Guangzhou University of Chinese Medicine (XK2019028).

\section{Footnote}

Reporting Checklist: The authors have completed the ARRIVE reporting checklist. Available at https://atm. amegroups.com/article/view/10.21037/atm-21-6742/rc

Data Sharing Statement: Available at https://atm.amegroups. com/article/view/10.21037/atm-21-6742/dss
Conflicts of Interest: All authors have completed the ICMJE uniform disclosure form (available at https://atm. amegroups.com/article/view/10.21037/atm-21-6742/coif). All authors report that this study was jointly funded by the National Natural Science Foundation of China (81973886) and Projects of Guangzhou University of Chinese Medicine (XK2019028). The authors have no other conflicts of interest to declare.

Ethical Statement: The authors are accountable for all aspects of the work in ensuring that questions related to the accuracy or integrity of any part of the work are appropriately investigated and resolved. All animal-related protocols were formulated based on the Guide for the Care and Use of Laboratory Animals by the National Research Council and approved by the Animal Care and Use Committee of Guangzhou University (No. SCXK2018-0034).

Open Access Statement: This is an Open Access article distributed in accordance with the Creative Commons Attribution-NonCommercial-NoDerivs 4.0 International License (CC BY-NC-ND 4.0), which permits the noncommercial replication and distribution of the article with the strict proviso that no changes or edits are made and the original work is properly cited (including links to both the formal publication through the relevant DOI and the license). See: https://creativecommons.org/licenses/by-nc-nd/4.0/.

\section{References}

1. Cannata-Andía JB, Rodriguez García M, Gómez Alonso C. Osteoporosis and adynamic bone in chronic kidney disease. J Nephrol 2013;26:73-80.

2. Pepe J, Body JJ, Hadji P, et al. Osteoporosis in Premenopausal Women: A Clinical Narrative Review by the ECTS and the IOF. J Clin Endocrinol Metab 2020;105:dgaa306.

3. Michaelson MD, Smith MR. Bisphosphonates for treatment and prevention of bone metastases. J Clin Oncol 2005;23:8219-24.

4. Huang D, Hou X, Zhang D, et al. Two novel polysaccharides from rhizomes of Cibotium barometz promote bone formation via activating the BMP2/SMAD1 signaling pathway in MC3T3-E1 cells. Carbohydr Polym 2020;231:115732.

5. Zhang ND, Han T, Huang BK, et al. Traditional Chinese medicine formulas for the treatment of osteoporosis: Implication for antiosteoporotic drug discovery. J 
Ethnopharmacol 2016;189:61-80.

6. Wang Y, Zhang L, Du Z, et al. Chemical Diversity and Prediction of Potential Cultivation Areas of Cistanche Herbs. Sci Rep 2019;9:19737.

7. Chen Y, Li YQ, Fang JY, et al. Establishment of the concurrent experimental model of osteoporosis combined with Alzheimer's disease in rat and the dual-effects of echinacoside and acteoside from Cistanche tubulosa. J Ethnopharmacol 2020;257:112834.

8. Li Z, Lin H, Gu L, et al. Herba Cistanche (Rou CongRong): One of the Best Pharmaceutical Gifts of Traditional Chinese Medicine. Front Pharmacol 2016;7:41.

9. Morikawa T, Xie H, Pan Y, et al. A Review of Biologically Active Natural Products from a Desert Plant Cistanche tubulosa. Chem Pharm Bull (Tokyo) 2019;67:675-89.

10. Xu X, Zhang Z, Wang W, et al. Therapeutic Effect of Cistanoside A on Bone Metabolism of Ovariectomized Mice. Molecules 2017;22:197.

11. Glick D, Barth S, Macleod KF. Autophagy: cellular and molecular mechanisms. J Pathol 2010;221:3-12.

12. Wan Y, Zhuo N, Li Y, et al. Autophagy promotes osteogenic differentiation of human bone marrow mesenchymal stem cell derived from osteoporotic vertebrae. Biochem Biophys Res Commun 2017;488:46-52.

13. Mauthe M, Orhon I, Rocchi C, et al. Chloroquine inhibits autophagic flux by decreasing autophagosome-lysosome fusion. Autophagy 2018;14:1435-55.

14. Patel MS, Karsenty G. Regulation of bone formation and vision by LRP5. N Engl J Med 2002;346:1572-4.

15. Esen E, Chen J, Karner CM, et al. WNT-LRP5 signaling induces Warburg effect through mTORC2 activation during osteoblast differentiation. Cell Metab 2013;17:745-55.

16. Zhong Z, Zylstra-Diegel CR, Schumacher CA, et al. Wntless functions in mature osteoblasts to regulate bone mass. Proc Natl Acad Sci U S A 2012;109:E2197-204.

17. Zhou R, Yuan Z, Liu J, et al. Calcitonin gene-related peptide promotes the expression of osteoblastic genes and activates the WNT signal transduction pathway in bone marrow stromal stem cells. Mol Med Rep 2016;13:4689-96.

18. Komori T. Regulation of bone development and extracellular matrix protein genes by RUNX2. Cell Tissue Res 2010;339:189-95.

19. MacDonald BT, He X. Frizzled and LRP5/6 receptors for Wnt/ $\beta$-catenin signaling. Cold Spring Harb Perspect Biol 2012;4:a007880.

20. Fu Z, Fan X, Wang X, et al. Cistanches Herba: An overview of its chemistry, pharmacology, and pharmacokinetics property. J Ethnopharmacol 2018;219:233-47.

21. Luo H, Cao R, Wang L, et al. Protective effect of Cistanchis A on ethanol-induced damage in primary cultured mouse hepatocytes. Biomed Pharmacother 2016;83:1071-9.

22. Li Z, Wang W, Xu H, et al. Effects of altered CXCL12/ CXCR4 axis on BMP2/Smad/Runx2/Osterix axis and osteogenic gene expressions during osteogenic differentiation of MSCs. Am J Transl Res 2017;9:1680-93.

23. Sharma U, Pal D, Prasad R. Alkaline phosphatase: an overview. Indian J Clin Biochem 2014;29:269-78.

24. Yang JX, He YB, Lai LN, et al. Electrochemical sensors using gold submicron particles modified electrodes based on calcium complexes formed with alizarin red $\mathrm{S}$ for determination of $\mathrm{Ca}(2+)$ in isolated rat heart mitochondria. Biosens Bioelectron 2015;66:417-22.

25. Dikic I, Elazar Z. Mechanism and medical implications of mammalian autophagy. Nat Rev Mol Cell Biol 2018;19:349-64.

26. Zhang L, Guo YF, Liu YZ, et al. Pathway-based genomewide association analysis identified the importance of regulation-of-autophagy pathway for ultradistal radius BMD. J Bone Miner Res 2010;25:1572-80.

27. Filomeni G, De Zio D, Cecconi F. Oxidative stress and autophagy: the clash between damage and metabolic needs. Cell Death Differ 2015;22:377-88.

28. Nollet M, Santucci-Darmanin S, Breuil V, et al. Autophagy in osteoblasts is involved in mineralization and bone homeostasis. Autophagy 2014;10:1965-77.

29. Thomas N, Choi HK, Wei X, et al. Autophagy Regulates Craniofacial Bone Acquisition. Calcif Tissue Int 2019;105:518-30.

30. Li H, Li D, Ma Z, et al. Defective autophagy in osteoblasts induces endoplasmic reticulum stress and causes remarkable bone loss. Autophagy 2018;14:1726-41.

31. Gordon MD, Nusse R. Wnt signaling: multiple pathways, multiple receptors, and multiple transcription factors. J Biol Chem 2006;281:22429-33.

32. Wei L, Ding L, Mo MS, et al. Wnt3a protects SH-SY5Y cells against 6-hydroxydopamine toxicity by restoration of mitochondria function. Transl Neurodegener 2015;4:11.

33. Chong ZZ, Shang YC, Hou J, et al. Wnt1 neuroprotection translates into improved neurological function during oxidant stress and cerebral ischemia through AKT1 and mitochondrial apoptotic pathways. Oxid Med Cell Longev 2010;3:153-65.

34. Pérez-Plasencia C, López-Urrutia E, García-Castillo V, 
et al. Interplay Between Autophagy and Wnt/B-Catenin Signaling in Cancer: Therapeutic Potential Through Drug Repositioning. Front Oncol 2020;10:1037.

35. Petherick KJ, Williams AC, Lane JD, et al. Autolysosomal $\beta$-catenin degradation regulates Wnt-autophagy-p62 crosstalk. EMBO J 2013;32:1903-16.

36. Fan Q, Yang L, Zhang X, et al. Autophagy promotes

Cite this article as: Chen T, Gao F, Luo D, Wang S, Zhao Y, Liu S, Huang J, Lin Y, Zhang Z, Huang H, Wan L. Cistanoside A promotes osteogenesis of primary osteoblasts by alleviating apoptosis and activating autophagy through involvement of the Wnt/ $\beta$-catenin signal pathway. Ann Transl Med 2022;10(2):64. doi: $10.21037 /$ atm-21-6742 metastasis and glycolysis by upregulating MCT1 expression and $\mathrm{Wnt} / \beta$-catenin signaling pathway activation in hepatocellular carcinoma cells. J Exp Clin Cancer Res 2018;37:9.

37. Hoverter NP, Waterman ML. A Wnt-fall for gene regulation: repression. Sci Signal 2008;1:pe43. 